\title{
The status of the critically endangered freshwater crayfish Engaewa pseudoreducta (Crustacea: Parastacidae) in south-western Australia
}

\author{
Quinton Burnham, Annette Koenders and Pierre Horwitz
}

\begin{abstract}
Centre for Ecosystem Management, School of Natural Sciences, Edith Cowan University, 270 Joondalup Drive, Joondalup, Western Australia 6027, Australia.
\end{abstract}

\begin{abstract}
An update of taxonomic, distribution and conservation information for the freshwater crayfish Engaewa pseudoreducta Horwitz and Adams is presented. Engaewa pseudoreducta is formally listed as Critically Endangered by the state of Western Australia and under Australian national legislature, and informally in a recently completed international (IUCN Red List) global assessment of freshwater crayfish. Engaewa pseudoreducta was first described on the basis of morphological characters of a small number of individuals from a single site, however the type locality was known to no longer support a population at the time of description and only one other nearby population was known prior to this study. This paper records two further populations and presents the first genetic analysis of the species. DNA sequence data support the recognition of E. pseudoreducta as a species and despite the discovery of additional populations there appears no reason to lessen the conservation concern surrounding the species, particularly in light of continuing development in the area.
\end{abstract}

KEYWORDS: Engaewa, conservation, molecular systematics, freshwater crayfish, 16S, south-western Australia

\section{INTRODUCTION}

The first scientific collections of obligate burrowing freshwater crayfish in south-western Australia were made in the late 1950s and, following subsequent collections in the early part of the next decade, Riek (1967) described three species, each from a single locality, and placed them within a new genus Engaewa Riek, 1967. As part of wide scale revisions of species in the freshwater crayfish family Parastacidae in Australia throughout the 1980s and 1990s (e.g. Austin 1986, 1996; Morgan 1986, 1988, 1997; Horwitz 1990; Horwitz, Adams and Baverstock 1990; Zeidler and Adams 1990; Austin and Knott 1996), Horwitz and Adams (2000) reviewed the status of the genus Engaewa. In doing so they described an additional two species (E. pseudoreducta Horwitz and Adams, 2000 and E. walpolea Horwitz and Adams, 2000) and proposed the first realistic species boundaries, after Riek (1967) initially suggested species boundaries of the three original species (E. reducta Riek, 1967, E. similis Riek, 1967 and E. subcoerulea Riek, 1967) based on collections from only four localities.

Horwitz and Adams (2000) recorded the occurrence of 41 populations across all five species (E. pseudoreducta - 1; E. reducta - 5; E. similis - 19; E. subcoerulea -
12; E. walpolea - 4) (based on their distribution map). All species in the genus show narrow geographical distributions with E. pseudoreducta being the quintessential example - its entire known range prior to this study constituted significantly less than $3 \mathrm{~km}^{2}$. The species was originally described from a collection made in 1985 in a clay-based wetland at the headwaters of a stream, some $15 \mathrm{~km}$ ENE of Margaret River. This site became the type locality for the species, but has subsequently been converted to a farm dam. The local catchment was converted to a blue gum (Eucalyptus globulus) plantation. Concern for Engaewa pseudoreducta was raised by Horwitz and Adams (2000) since it could no longer be found at the type locality following the modification of habitat and had only been found at one other site in the next drainage line to the west. Engaewa pseudoreducta was subsequently gazetted on Schedule 1 (Fauna that is rare or is likely to become extinct) (Wildlife Conservation (Specially Protected Fauna) Notice 2006), under the Western Australian Wildlife Conservation Act 1950, on the criteria that it had very restricted areas of occurrence and occupancy, with extreme fluctuations in area, extent and/or quality of habitat, and number of locations or subpopulations.

Recovery planning by the State's Department of 


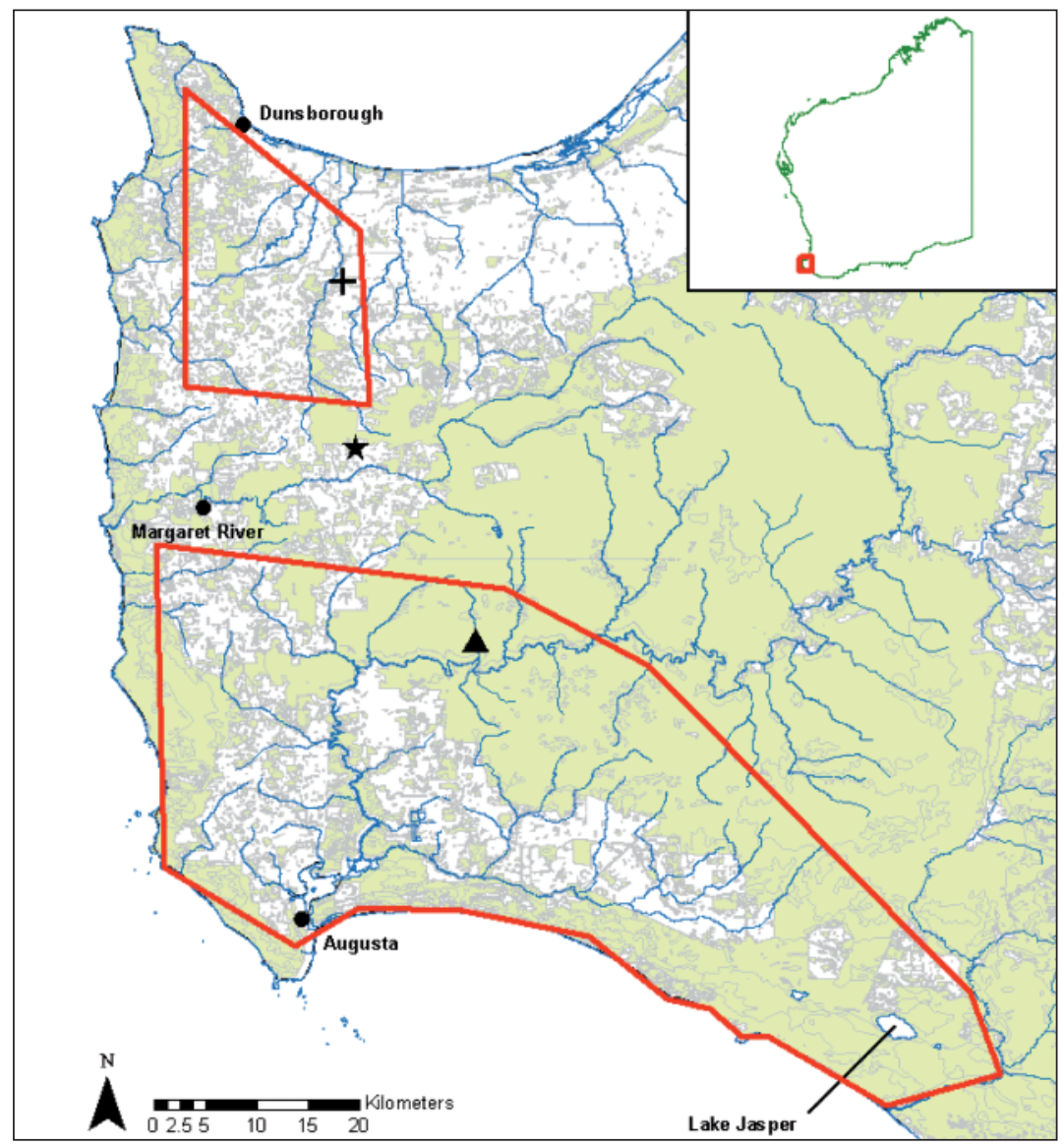

FIGURE 1 Location of important populations for this study (showing known E. pseudoreducta population (star), a new population at Payne Rd (cross), and Clade A population (triangle). Also shown are the approximated distribution polygons for E. reducta (northern) and E. similis (southern) as considered by Riek (1967) and Horwitz and Adams (2000). The search area for this study encompassed suitable habitat between Dunsborough and Lake Jasper.

Environment and Conservation commenced in 2007 for this species (and two others in the genus listed as threatened), during which time nominations were prepared for federal recognition and in 2009 the species was gazetted as Critically Endangered under the Commonwealth of Australia's EPBC Act (1999). Recovery operations also included an extensive survey of the geographical range of the genus by the lead author, in the hope that additional populations of E. pseudoreducta could be found, and to determine the genetic variability between this species and others in the genus, for any populations found. This paper presents the findings of these investigations and discusses their significance in view of the conservation status of the species.

\section{MATERIALS AND METHODS}

As Engaewa spend virtually their entire life below ground, one immediate challenge is to confirm their presence at a site. In order to direct collection efforts, in the first instance potential habitat was identified using a combination of maps and satellite imagery, looking for small creeks or potentially larger swamp systems that possessed a significant canopy of native vegetation. This approach created a list of potential habitat sites that required ground-truthing. Areas that appeared to be suitable from maps and imagery were often not so once visited and vice versa, hence the only reliable way to assess an area was to visit it. As such, virtually every accessible creek, drainage line, swamp or seepage with some degree of native vegetation remaining within the search area was examined.

An important corollary here is that non-detection of Engaewa at a particular site cannot be taken as definitive evidence of its absence (i.e. a false negative), which is true of all presence-absence records (MacKenzie 2005). However, significant limitations to the likelihood of detection notwithstanding intense survey effort are especially pertinent for Engaewa, and of great importance for a critically endangered species such as E. pseudoreducta. Notable impediments to detection of Engaewa are the species' cryptic, burrowing nature, the difficulty seeing and accessing burrows in often dense vegetation and gaining access (by road) to potential sites during the wet season when the animals are most active and the soil is suitable for digging. 
For Engaewa pseudoreducta the search area was defined as being between Dunsborough and Lake Jasper, east of Augusta (Figure 1), a distance of approximately $150 \mathrm{~km}$ following the coastline and extending $40 \mathrm{~km}$ inland. This is far beyond its known distribution and also encompasses the ranges of two other species considered, with E. pseudoreducta, to represent a species-complex in the genus (Horwitz and Adams 2000). All areas of potentially suitable habitat occurring on Crown Lands were examined, as well as a number of sites located on private property throughout the assumed potential distribution of the species. On site, the presence of Engaewa species was indicated by the occurrence of 'chimneys' of pelleted soil at the entrance to a burrow system (Figure 2). These piles of soil are formed when material is expelled from the burrows dug by the crayfish. The individual pellets can be up to 1-2 $\mathrm{cm}$ in diameter, though generally are much smaller, and the chimney can range from less than half a dozen small pellets surrounding a small hole to a conical shaped chimney up to $35 \mathrm{~cm}$ high and formed from tens or even hundreds of individual pellets. The soil forming the chimney may be distinctly pelleted or it may appear as a simple pile of soil, due to the effect of weathering. Where obvious chimneys were lacking, closer attention was paid to any patches of different coloured soil or even simple holes in the ground that may also signal the entrance to a burrow should the chimney have weathered away entirely.

The presence of chimneys is not definitive evidence of inhabitation by Engaewa species because members of the freshwater crayfish genus Cherax also construct chimneys in particular soil types. An experienced surveyor, however, can usually recognise slight variations in the characteristics of the chimneys produced by these different crayfish. Engaewa chimneys are usually far more substantial and the pellets of soil much smaller. Cherax species typically dig short, straight tunnels and, as such, have small chimneys with much larger pellets due to their larger body size. Cherax chimneys also often form a miniature caldera, whereas Engaewa chimneys almost always appear conical. The diameter of the tunnel extending vertically from the chimney is also characteristic as Engaewa burrows are much smaller in diameter (approximately a 'pinky' finger in width) when compared to a Cherax burrow (often in the range of middle finger to thumb in width and, at times, larger). Once a potential Engaewa species burrow had been identified, it was excavated, and if crayfish belonging to the genus Engaewa were found, they were collected for morphological and molecular analyses.

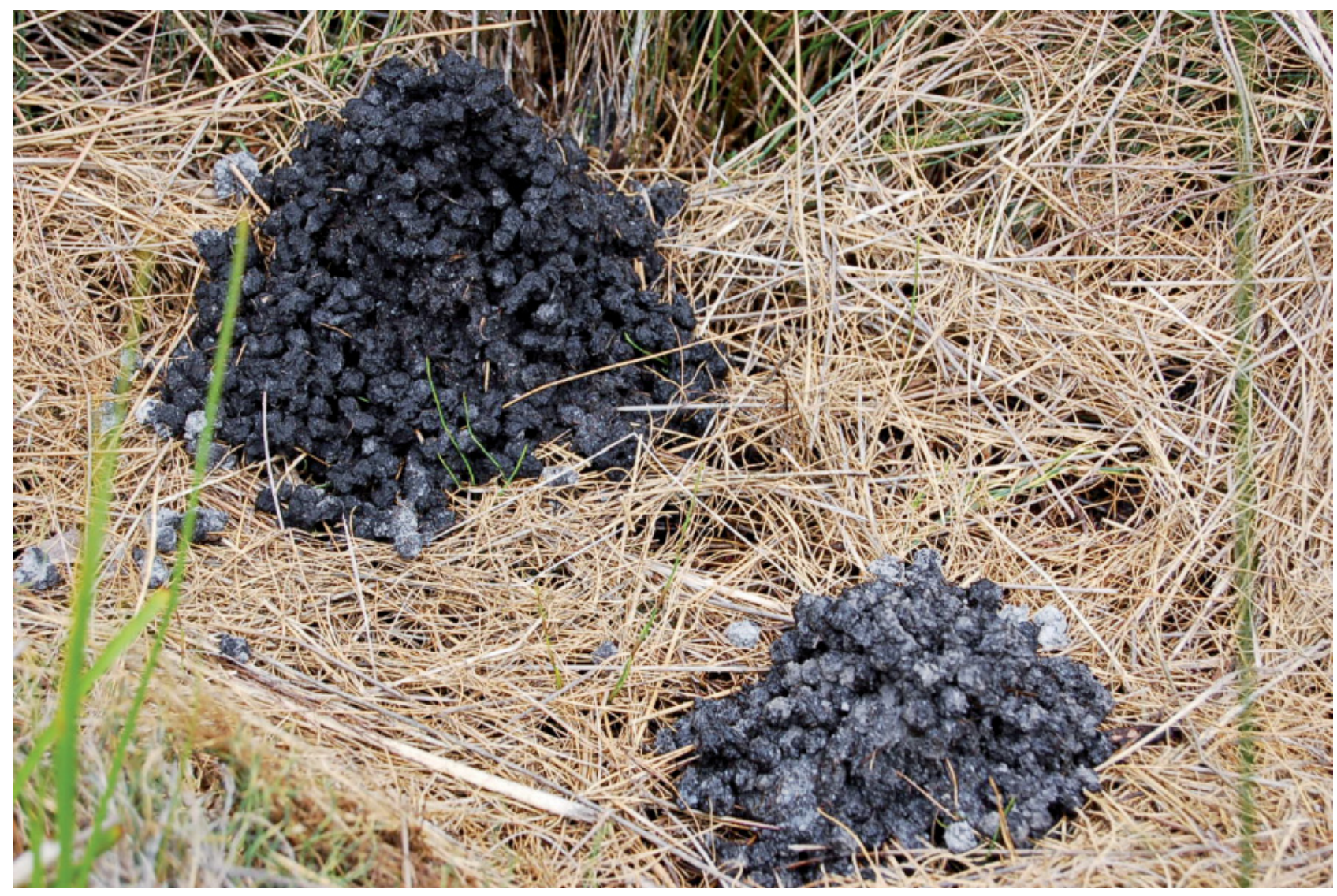

FIGURE 2 Two 'chimneys', formed by spherical pellets of soil, which indicate the entrance to an Engaewa burrow system. 
Crayfish were initially identified according to the diagnostic morphological characters described by Horwitz and Adams (2000) (for E. pseudoreducta the most easily recognised diagnostic characters were the presence of patches of setae on the ventral surface of the merus, ventrally and distally on the carpus, laterally adjacent to cutting edges, and occasionally on the propodal palm as well). Genetic characterization was then undertaken to confirm or refute the species descriptions of Horwitz and Adams (2000).

DNA was isolated using the DNeasy Blood and Tissue Kit (QIAGEN) from tail or gill tissue of ethanol preserved specimens. PCR was used to amplify part of the mitochondrial large rDNA (16S rDNA), using total genomic DNA as a template, and primers 1471 (5'-CCTGTTTANCAAAAACAT-3') and 1472 (5'AGATAGAAACCAACCTGG-3') (Crandall, Lawler and Austin 1995; Crandall and Fitzpatrick 1996), and HotStarTaq Plus Master Mix (QIAGEN). The cycling conditions were an initial denaturing step $\left(94^{\circ} \mathrm{C}\right.$ for $\left.5 \mathrm{~min}\right), 35$ cycles of denaturing $\left(94^{\circ} \mathrm{C}\right.$ for $30 \mathrm{sec})$, annealing $\left(46^{\circ} \mathrm{C}\right.$ for $\left.30 \mathrm{sec}\right)$ and extension $\left(72^{\circ} \mathrm{C}\right.$ for $\left.45 \mathrm{sec}\right)$, and a final extension step $\left(72^{\circ} \mathrm{C}\right.$ for $7 \mathrm{~min}$ ). PCR products were sent to Macrogen Inc. (Seoul, South Korea) to be purified and sequenced automatically and directly using the ABI BigDye chemistry. Chromatograms were checked manually and edited by comparing the sequence derived from forward and reverse primers in FinchTV v.1.4 (http://www. geospiza.com/Products/finchtv.shtml). The consensus sequences were then aligned using MUSCLE (Edgar 2004) as implemented in MEGA5 (Tamura et al. 2011).

The phylogenetic relationships between specimens were inferred using Maximum Likelihood (ML) analyses, conducted in PhyML v2.4.4 (Guindon and Gascuel 2003) based on the best substitution model selected by jModeltest v3.7 (Posada 2008) under the Akaike Information Criterion (Akaike 1974) with support for nodes assessed by non-parametric bootstrap (Felsenstein 1985) with 1000 bootstrap replicates. The non-rooted bootstrap tree was visualised with Figtree v.1.3.1 (http://tree.bio.ed.ac.uk/software/figtree/). In order to further discuss species boundaries we calculated genetic distances within and between clades identified in this study and Engaeus Erichson, 1846 using maximum composite likelihood in MEGA5 (Tamura et al. 2011). Following Schultz et al. (2009) the genus Engaeus was viewed as two distinct clades, Engaeus sensu stricto and Engaeus lyelli.

The appropriate conservation status of Engaewa pseudoreducta was assessed based on the current IUCN/ EPBC criteria. Areas of occurrence and occupancy for E. pseudoreducta were calculated by drawing polygons in Google Earth (version 6.0.1.2032 beta) and then the saved KML files were entered into the University of New Hampshire Cooperative Extension Tools web-based polygon program (http://extension.unh.edu/kmlTools/ index.cfm).

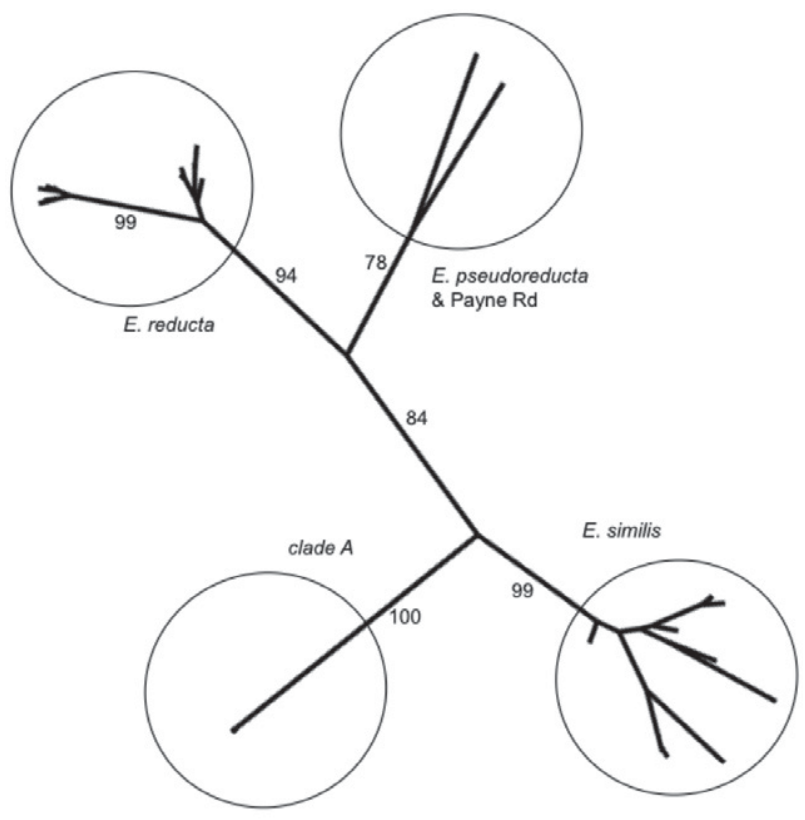

FIGURE 3 Unrooted ML tree for 47 specimens of Engaewa constructed from 394 basepairs of the $16 \mathrm{~S} \mathrm{mtDNA}$, with boostrap support (1000 replicates) shown for the major branches in the tree. Clear molecular support for four clades identified by morphological characters (E. pseudoreducta (including the newly uncovered population at Payne Road), E. reducta, E. similis and Clade $A$ ) is evident from this tree.

\section{RESULTS}

Engaewa were collected from 26 sites throughout the study area and 47 specimens from these sites were analysed for this review. The specimens collected were deposited in the Western Australian Museum (Appendix 1). Based on the diagnostic morphological characters defined by Horwitz and Adams (2000), 22 specimens from 11 sites belong to $E$. similis, 17 specimens from 12 sites to $E$. reducta, one specimen belongs to E. pseudoreducta and two specimens (referred to as 'Payne Road') were morphologically consistent with the description of E. pseudoreducta, but occurred outside of its known distribution, while five specimens from a single locality had indeterminate character states (A full review of the genus is being prepared as part of the $\mathrm{PhD}$ thesis of the senior author.). The 47 new partial mitochondrial $16 \mathrm{~S}$ rDNA nucleotide sequences were deposited on NCBI GenBank (accession numbers JQ613107 to 613153), whilst 23 Engaeus sequences from NCBI Genbank were included (19 Engaeus sensu stricto and 4 Engaeus lyelli) (Appendix 1).

The 16S analysis is presented as an unrooted ML tree showing that all specimens defined on the basis of their morphology also form monophyletic genetic 
groups (Figure 3). Although not presented here, the same groupings are supported by other mitochondrial markers as well as nuclear markers - these data will be presented in the upcoming full review of the genus. The single specimen assumed to represent E. pseudoreducta and the specimens from 'Payne Road' are clearly divergent from all other species, are each others' nearest relatives and form a distinct clade. A fourth clade (referred to herein as Clade A) is formed solely by the representatives alluded to above as showing indeterminate character states.

With the exception of Clade $A$ (representing a single population), each morphologically defined species shows at least two distinct genetic lineages within their respective clade (Figure 3). These genetic lineages correspond strongly to geographic partitioning of populations and are responsible for the relatively high genetic distances within these species (Table 1). This is particularly evident for the reducta and similis clades, which are relatively widespread (on the scale of Engaewa distribution), however, the significance of the relatively high genetic divergence between the two populations in the pseudoreducta clade (Figure 3 , Table 1) is difficult to interpret without additional samples, either from the populations in question or any other populations that may be genetically similar (if any exist). Nevertheless the few specimens collected from these two sites do conform closely to the morphological description of the species E. pseudoreducta presented by Horwitz and Adams (2000).

Maximum composite likelihood genetic distances (Table 1) between Engaewa clades and Engaeus sensu stricto ranged from 0.294-0.324 and between Engaewa clades and Engaeus lyelli 0.369-0.396. In contrast, the distance between Engaeus sensu stricto and Engaeus lyelli was 0.279 . The distances between Engaeus sensu stricto species ranged from 0.017-0.302 (data not shown) and those for Engaewa clades were in the middle of this range at 0.131-0.175. Within clade genetic distances were 0.172 for Engaeus sensu stricto, 0.035 for Engaeus lyelli and $\sim 0.065$ for Engaewa with the exception of Engaewa Clade A, representing a single population with a value of 0.003 .

The IUCN Red List Categories and Criteria: Version 3.1. (2001) states that "A taxon is Critically Endangered when the best available evidence indicates that it ... [is] considered to be facing an extremely high risk of extinction in the wild". The geographic range of this species, both in terms of the extent of occurrence and area of occupancy (criteria B1 and B2), falls well within the criteria for the category of Critically Endangered $\left(<100 \mathrm{~km}^{2}\right.$ and $<10 \mathrm{~km}^{2}$ respectively). Furthermore, to fully satisfy criterion B2 and thus be validly considered as Critically Endangered, the species in question must conform to at least two of three further requirements. Whilst E. pseudoreducta are no longer believed to exist at only a single location there is no doubt that the distribution of populations is severely fragmented (preventing a metapopulation scenario whereby migrants can replace any localised extinctions), thus satisfying criterion $\mathrm{B} 2 \mathrm{a}$, and the loss of the largest known population at the type locality satisfies criterion B2b (an observed decline of extent of occurrence, area of occupancy, area, extent and/or quality of habitat, number of locations or subpopulations and number of mature individuals). The degree to which the natural environment has been altered since European colonization, and the resulting severe habitat fragmentation, can be easily inferred from the image in Figure 1. It can be reasonably argued that all of these can be 'inferred or projected' based on past and on-going anthropogenic impacts in the area, particularly when combined with suggested impacts of future climate change for the region (Horwitz et al. 2008).

TABLE 1 Composite maximum likelihood genetic distances (16S). Values on the diagonal (bold) represent the distances within species (standard errors $<0.02$ ), those below the diagonal are distances between clades and those above the diagonal (italicised) are the associated standard errors.

\begin{tabular}{|c|c|c|c|c|c|c|c|c|}
\hline $\mathrm{N}$ & Clade & & [1] & [2] & [3] & [4] & {$[5]$} & [6] \\
\hline 3 & Engaewa pseudoreducta & [1] & 0.065 & 0.024 & 0.027 & 0.026 & 0.046 & 0.067 \\
\hline 17 & Engaewa reducta & [2] & 0.175 & 0.065 & 0.025 & 0.026 & 0.051 & 0.068 \\
\hline 22 & Engaewa similis & [3] & 0.153 & 0.161 & 0.068 & 0.022 & 0.047 & 0.067 \\
\hline 5 & Engaewa Clade A & [4] & 0.156 & 0.164 & 0.131 & 0.003 & 0.046 & 0.067 \\
\hline 19 & Engaeus sensu stricto & {$[5]$} & 0.312 & 0.324 & 0.304 & 0.294 & 0.172 & 0.045 \\
\hline 4 & Engaeus lyelli & [6] & 0.386 & 0.396 & 0.387 & 0.369 & 0.279 & 0.035 \\
\hline
\end{tabular}


TABLE 2 Locality and collection details for all known specimens of E. pseudoreducta.

$\begin{array}{lll}\text { Locality } & \begin{array}{l}\text { Year collection } \\ \text { made }\end{array} & \begin{array}{l}\text { Specimens } \\ \text { collected }\end{array}\end{array} \quad$ Site description and notes

\begin{tabular}{|c|c|c|c|}
\hline $\begin{array}{l}\text { "... near Osmington, } \\
\text { north-east of Margaret } \\
\text { River" } 1\end{array}$ & 1985 & $\begin{array}{l}5 \text { adults } \\
5 \text { juveniles }\end{array}$ & $\begin{array}{l}\text { "... burrows around a recently constructed farm dam, in the } \\
\text { middle of a swamp with remnant vegetation consisting of tall } \\
\text { ti-trees and some eucalypts; the soil in the area was a yellow- } \\
\text { light brown silty sandy clay; in undisturbed parts of the small } \\
\text { swamp, burrows ramified laterally just below the surface, and } \\
\text { water was found in pools at the surface in August } 1985 \text {. This } \\
\text { site has undergone substantial change since then." }\end{array}$ \\
\hline
\end{tabular}

Treeton Reserve Site $12003 \quad 1$ juvenile Burrows excavated in broad section of a densely-vegetated creek line, with high water levels, in November.

Treeton Reserve Site $2007 \quad 1$ adult

Payne Rd $2007 \quad 2$ adults

"...found in the heavy clay soils of narrow valleys in and adjoining Treeton reserve. The burrows found during this study were identified by small piles of slightly different coloured soil. This soil is likely to have represented washed down chimneys as there had been significant rainfall and the burrows were within a small creek line. As the water table was so high at the time of collecting this species the burrow systems were not fully explored though they appeared to branch laterally at a shallow depth as well as possessing tunnels proceeding deeper." 2

Burrows indicated by partially weathered sandy chimneys. Crayfish dug out of coarse sand in a broad, flat area with sluggish and intertwined shallow draining channels with the water table just below ground level.

${ }^{1}$ From Horwitz and Adams (2000). ${ }^{2}$ From Burnham et al. (2007).

\section{DISCUSSION}

Our findings suggest that Engaewa pseudoreducta should be recognised as a valid species on the basis of morphological and molecular (DNA sequence) data. In fact, morphological characters and DNA sequence data support the recognition of all three current species of Engaewa (E. pseudoreducta, E. similis and E. reducta) within the northern part of the range of the genus (roughly bounded by the region searched in this study) and suggest that an additional species (Clade $A$ ) should be recognised (species descriptions for this candidate species and another from the southern part of Engaewa's range are currently being undertaken by the lead author). Prior to this study it had been recognised that a specific site within the study region contained "errant specimens [that] warrant closer examination" (Horwitz and Adams 2000, p. 677) and Clade A corresponds to the population referred to in that publication.

A comparison within and between the Engaewa species included in this study and a number of species from the closely related genus Engaeus (considered as two distinct clades Engaeus sensu stricto and Engaeus lyelli following Schultz et al., 2009) is made using 16S genetic distances. The pairwise distances between Engaewa, Engaeus sensu stricto and Engaeus lyelli are similar and support the presence of three clades, with Engaeus sensu stricto and Engaeus lyelli more similar to each other than either to Engaewa. The genetic distances within Engaewa clades had low variation (except Clade $A$ which is from a single population and shows low diversity) and the distances between Engaewa clades are in the middle of the range for Engaeus sensu stricto. The genetic distances therefore support the current Engaewa molecular species groupings and morphological species descriptions, whilst also indicating the presence of an additional, undescribed species (Clade $A$ ).

Considering the conservation concern regarding Engaewa pseudoreducta, the dearth of specimens/ populations to study and the lack of diagnostic morphological characters to distinguish between $E$. pseudoreducta specimens from the one drainage line which includes the type locality, and the specimen at Payne Road, it seems prudent to treat them as representatives of a single species until such time as the treatment of further specimens and/or other analyses can be undertaken. The genetic divergence between these 
two samples hints at the possibility that interpopulation mtDNA diversity is extremely high for E. pseudoreducta (and the forthcoming review of the genus suggests that this is generally true for Engaewa as a whole); thus, making these existing populations even more significant from a conservation viewpoint. We therefore suggest that the currently acknowledged geographic range of E. pseudoreducta should include the drainage system from which the original description was made and be extended to include the population at Payne Road, some $16 \mathrm{~km}$ north (details of the sites from which $E$. pseudoreducta have been collected are presented in Table 2). We also note the presence of both E. similis and E. reducta at several sites very close to the drainage lines inhabited by E. pseudoreducta, though they have never been found in sympatry.

Including the population at Payne Road increases E. pseudoreducta's known range twenty-fold, to a region of about $60 \mathrm{~km}^{2}$. However, this figure somewhat misrepresents the distribution of the species; once unsuitable habitat is removed, the potential area of occupancy is probably less than $2.5 \mathrm{~km}^{2}$ and the actual area of occupancy may well be significantly less again. Additional sampling should continue in the region in the hope of closing the geographic gap between the $E$. pseudoreducta populations. As noted above, Engaewa are highly cryptic due to their small size and almost exclusively subterranean existence, which makes the task of confirming their absence from potential habitat difficult. While we are confident that we have searched extensively, it is not impossible that one or more isolated, small populations remain undetected in this fragmented landscape.

The conservation status of Critically Endangered assigned to E. pseudoreducta under the EPBC Act can be re-examined using the data presented in this paper (and indeed this has been done for a recent review of the conservation status of all freshwater crayfish conducted for the IUCN Red List). The discovery of additional populations potentially bodes well for the survival of the species as a whole, although an increase from one to three populations is obviously not a reason to reduce concern, particularly as downstream habitat alteration has isolated all populations into small pockets of suitable habitat.

The inland aquatic biodiversity of south-western Australia, particularly in the coastal margins, is facing significant and increasing survival pressure due to large-scale human endeavours (Horwitz et al. 2008). Agriculture, urbanization, groundwater extraction and mining have all altered the natural character of the region. These changes increase the vulnerabilities of a species such as E. pseudoreducta, which appears to possess low dispersal ability, is wedded to highly restricted and disjunct habitat, and persists in only a few isolated populations. Based on our data we conclude that the conservation status of E. pseudoreducta (Critically
Endangered) should remain unchanged.

\section{ACKNOWLEDGEMENTS}

This paper presents part of a larger project funded by the Department of Environment and Conservation (DEC) through the South-West Catchment Council and the School of Natural Sciences and Centre for Ecosystem Management at Edith Cowan University, while the senior author was in receipt of an Australian Postgraduate Award. Kelly Burnham, Kellie Mantle and Michael Howie all provided considerable assistance in undertaking field collections of specimens and Kellie Mantle assisted in researching potential collecting sites. Collections were made under permit issued by (DEC). Isa Schön provided technical assistance with the molecular methodology employed in this study.

\section{REFERENCES}

Akaike, H. (1974). A new look at the statistical model identification. IEEE Transactions on Automatic Control 19: 716-723.

Austin, C.M. (1986). Electrophoretic and morphological systematic studies of the genus Cherax (Decapoda: Parastacidae) in Australia. PhD Thesis. (Department of Zoology, University of Western Australia: Perth.)

Austin, C.M. (1996). Systematics of the freshwater crayfish genus Cherax Erichson (Decapoda: Parastacidae) in northern and eastern Australia: Electrophoretic and morphological variation. Australian Journal of Zoology 44: 259-296.

Austin, C.M. and Knott, B. (1996). Systematics of the freshwater crayfish genus Cherax Erichson (Decapoda: Parastacidae) in south-western Australia: electrophoretic, morphological and habitat variation. Australian Journal of Zoology 44: 223-258.

Burnham, Q., Koenders, A. and Horwitz, P. (2007). Field studies into the biology and conservation requirements of Engaewa species in the South West and Warren DEC regions. Final Report Prepared for the Department of Environment and Conservation, Perth.

Crandall, K.A., Lawler, S.H. and Austin, C.M. (1995). A preliminary examination of the molecular phylogenetic relationships of some crayfish genera from Australia (Decapoda: Parastacidae). Freshwater Crayfish 10: 18-30.

Crandall, K.A. and Fitzpatrick, J.F. (1996). Crayfish molecular systematics: using a combination of procedures to estimate phylogeny. Systematic Biology 45: 1-26.

Edgar, R.C. (2004). MUSCLE: multiple sequence alignment with high accuracy and high throughput. Nucleic Acids Research 32: 1792-1797.

Felsenstein, J. (1985). Confidence limits on phylogenies: an approach using the Bootstrap. Evolution 39: 783-791.

Guindon, S. and Gascuel, O. (2003). A simple, fast, and accurate algorithm to estimate large phylogenies by maximum likelihood. Systematic Biology 52: 696-704.

Horwitz, P. (1990). A taxonomic revision of species in the freshwater crayfish genus Engaeus Erichson (Decapoda: Parastacidae). Invertebrate Taxonomy 4: 427-614. 
Horwitz, P. and Adams, M. (2000) The systematics, biogeography and conservation status of the species in the freshwater crayfish genus Engaewa Riek (Decapoda: Parastacidae) from south-western Australia. Invertebrate Taxonomy 14: 655-680.

Horwitz, P., Adams, M. and Baverstock, P. (1990). Electrophoretic contributions to the systematics of the freshwater crayfish genus Engaeus Erichson (Decapoda: Parastacidae). Invertebrate Taxonomy 4: 615-641.

Horwitz, P., Bradshaw, D., Hopper, S.D., Davies, P.M., Froend, R. and Bradshaw, F. (2008). Hydrological change escalates risk of ecosystem stress in Australia's threatened biodiversity hotspot. Journal of the Royal Society of Western Australia 91: 1-11.

IUCN (2001). IUCN Red List Categories and Criteria: Version 3.1. IUCN Species Survival Commission. IUCN, Gland, Switzerland and Cambridge, UK.

MacKenzie, D.I. (2005). What Are the Issues with PresenceAbsence Data for Wildlife Managers? The Journal of Wildlife Management 69: 849-860.

Morgan, G.J. (1986). Freshwater crayfish of the genus Euastacus Clark (Decapoda, Parastacidae) from Victoria. Memoirs of the Museum of Victoria 47: 1-57.

Morgan, G.J. (1988). Freshwater crayfish of the genus Euastacus Clark (Decapoda, Parastacidae) from Queensland. Memoirs of the Museum of Victoria 49: 1-49.

Morgan, G.J. (1997). Freshwater crayfish of the genus
Euastacus Clark (Decapoda: Parastacidae) from New South Wales, with a key to all species of the genus. Records of the Australian Museum, Supplement 23: 1-110.

Posada, D. (2008). jModelTest: Phylogenetic Model Averaging. Molecular Biology and Evolution 25: 1253-1256.

Pybus, O.G. (2006). Model selection and the molecular clock. PloS Biology 4: 686-688.

Riek, E.F. (1967). The freshwater crayfish of Western Australia (Decapoda: Parastacidae). Australian Journal of Zoology 15: 103-21.

Schultz, M.B., Smith, S.A., Horwitz, P., Richardson, A.M.M., Crandall, K.A. and Austin, C.M. (2009). Evolution underground: A molecular phylogenetic investigation of Australian burrowing freshwater crayfish (Decapoda: Parastacidae) with particular focus on Engaeus Erichson. Molecular Phylogenetics and Evolution 50: 580-598.

Tamura, K., Peterson, D., Peterson, N., Stecher, G., Nei, M. and Kumar, S. (2011). MEGA5: Molecular Evolutionary Genetics Analysis using Maximum Likelihood, Evolutionary Distance, and Maximum Parsimony Methods. Molecular Biology and Evolution (submitted).

Zeidler, W. and Adams, M. (1990). Revision of the Australian crustacean genus of freshwater crayfish Gramastacus Riek (Decapoda : Parastacidae). Invertebrate Taxonomy 3: 913-924.

MANUSCRIPT RECEIVED 12 FEBRUARY 2011; ACCEPTED 10 FEBRUARY 2012. 
APPENDIX 1 Specimens used in this study and their GenBank accession numbers. All Engaewa sequences generated by the authors, whilst all Engaeus sequences were obtained from NCBI Genbank.

\begin{tabular}{|c|c|c|}
\hline Taxon & Sample Identification & GenBank Accession No. \\
\hline Engaewa pseudoreducta & WAM C49511 & JQ613110 \\
\hline Engaewa pseudoreducta & WAM C49512 & JQ613111 \\
\hline Engaewa pseudoreducta & WAM C49513 & JQ613118 \\
\hline Engaewa reducta & WAM C49514 & JQ613152 \\
\hline Engaewa reducta & WAM C49516 & JQ613153 \\
\hline Engaewa reducta & WAM C49517 & JQ613117 \\
\hline Engaewa reducta & WAM C49520 & JQ613148 \\
\hline Engaewa reducta & WAM C49521 & JQ613149 \\
\hline Engaewa reducta & WAM C49524 & JQ613150 \\
\hline Engaewa reducta & WAM C49525 & JQ613128 \\
\hline Engaewa reducta & WAM C49535 & JQ613130 \\
\hline Engaewa reducta & WAM C49537 & JQ613131 \\
\hline Engaewa reducta & WAM C49542 & JQ613125 \\
\hline Engaewa reducta & WAM C49543 & JQ613145 \\
\hline Engaewa reducta & WAM C49545 & JQ613127 \\
\hline Engaewa reducta & WAM C49547 & JQ613129 \\
\hline Engaewa reducta & WAM C49548 & JQ613132 \\
\hline Engaewa reducta & WAM C49549 & JQ613136 \\
\hline Engaewa reducta & WAM C49555 & JQ613109 \\
\hline Engaewa reducta & WAM C49558 & JQ613123 \\
\hline Engaewa similis & WAM C49560 & JQ613146 \\
\hline Engaewa similis & WAM C49561 & JQ613147 \\
\hline Engaewa similis & WAM C49562 & JQ613112 \\
\hline Engaewa similis & WAM C49565 & JQ613119 \\
\hline Engaewa similis & WAM C49566 & JQ613120 \\
\hline Engaewa similis & WAM C49567 & JQ613121 \\
\hline Engaewa similis & WAM C49568 & JQ613133 \\
\hline Engaewa similis & WAM C49570 & JQ613134 \\
\hline Engaewa similis & WAM C49571 & JQ613108 \\
\hline Engaewa similis & WAM C49573 & JQ613126 \\
\hline Engaewa similis & WAM C49575 & JQ613135 \\
\hline Engaewa similis & WAM C49577 & JQ613137 \\
\hline Engaewa similis & WAM C49578 & JQ613138 \\
\hline Engaewa similis & WAM C49579 & JQ613139 \\
\hline Engaewa similis & WAM C49580 & JQ613140 \\
\hline Engaewa similis & WAM C49581 & JQ613141 \\
\hline Engaewa similis & WAM C49582 & JQ613142 \\
\hline
\end{tabular}




\begin{tabular}{|c|c|c|}
\hline Taxon & Sample Identification & GenBank Accession No. \\
\hline Engaewa similis & WAM C49583 & JQ613114 \\
\hline Engaewa similis & WAM C49586 & JQ613115 \\
\hline Engaewa similis & WAM C49587 & JQ613116 \\
\hline Engaewa similis & WAM C49588 & JQ613122 \\
\hline Engaewa similis & WAM C49589 & JQ613124 \\
\hline Engaewa Clade A & WAM C49676 & JQ613144 \\
\hline Engaewa Clade A & WAM C49677 & JQ613151 \\
\hline Engaewa Clade A & WAM C49678 & JQ613107 \\
\hline Engaewa Clade A & WAM C49679 & JQ613113 \\
\hline Engaeus cisternarius & Voucher Museum Victoria J45407 & EF493110 \\
\hline Engaeus disjuncticus & Voucher Museum Victoria J45405 & EF493102 \\
\hline Engaeus fossor & Voucher Museum Victoria J45510 & EF493103 \\
\hline Engaeus fultoni & Voucher AIR2.1 & EF493042 \\
\hline Engaeus hemicirratulus & Voucher Museum Victoria J14750 & EF493104 \\
\hline Engaeus karnanga & Voucher Museum Victoria J45692 & EF493105 \\
\hline Engaeus laevis & Voucher LEL1.1 & EF493088 \\
\hline Engaeus lyelli & Voucher ENF1.2 & EF493073 \\
\hline Engaeus lyelli & Voucher Museum Victoria J14710 & EF493107 \\
\hline Engaeus lyelli & Museum Victoria J14711 & EF493108 \\
\hline Engaeus lyelli & Voucher NRN2.1 & EF493121 \\
\hline Engaeus mairener & Voucher Museum Victoria J45680 & EF493109 \\
\hline Engaeus mallacoota & Voucher Museum Victoria J14713 & EF493096 \\
\hline Engaeus martigener & Voucher Museum Victoria J45432 & EF493111 \\
\hline Engaeus merosetosus & Voucher WPC2.1 & EF493153 \\
\hline Engaeus nulloporius & Voucher Museum Victoria J4106 & EF493112 \\
\hline Engaeus orientalis & Voucher Museum Victoria J14725 & EF493113 \\
\hline Engaeus phyllocercus & Voucher Aus. Museum P67188 & EF493041 \\
\hline Engaeus sericatus & Voucher PEN1.4 & EF493125 \\
\hline Engaeus spinicaudatus & Voucher Museum Victoria J45696 & EF493114 \\
\hline Engaeus strictifrons & Voucher TWH1.1 & EF493149 \\
\hline Engaeus urostrictus & Voucher Museum Victoria J45681 & EF493115 \\
\hline Engaeus yabbimunna & Voucher Museum Victoria J34475 & EF493101 \\
\hline
\end{tabular}

